\title{
Comparison Between the Fundamental Group Scheme of a Relative Scheme and that of its Generic Fiber
}

\author{
MARCO ANTEI
}

November 20, 2018

\begin{abstract}
Résumé. On montre que le morphisme canonique $\varphi: \pi_{1}\left(X_{\eta}, x_{\eta}\right) \rightarrow \pi_{1}(X, x)_{\eta}$ entre le schéma en groupes fondamental de la fibre générique $X_{\eta}$ d'un schéma $X$ sur un schéma de Dedekind connexe et la fibre générique du schéma en groupes fondamental de $X$ est toujours fidèlement plat. On donnera ensuite des conditions nécessaires et suffisantes pour qu'un $G$-torseur fini, dominé et pointé au dessus de $X_{\eta}$ puisse être étendu sur $X$. On décrira des exemples où $\varphi: \pi_{1}\left(X_{\eta}, x_{\eta}\right) \rightarrow \pi_{1}(X, x)_{\eta}$ est un isomorphisme.
\end{abstract}

Abstract. We show that the natural morphism $\varphi: \pi_{1}\left(X_{\eta}, x_{\eta}\right) \rightarrow \pi_{1}(X, x)_{\eta}$ between the fundamental group scheme of the generic fiber $X_{\eta}$ of a scheme $X$ over a connected Dedekind scheme and the generic fiber of the fundamental group scheme of $X$ is always faithfully flat. As an application we give a necessary and sufficient condition for a finite, dominated pointed $G$-torsor over $X_{\eta}$ to be extended over $X$. We finally provide examples where $\varphi: \pi_{1}\left(X_{\eta}, x_{\eta}\right) \rightarrow$ $\pi_{1}(X, x)_{\eta}$ is an isomorphism.

\section{Introduction}

In [17 and 16] (respectively) Saïdi and Romagny give an example of a $G$ torsor $Y$ over the generic fiber $X_{\eta}$ of a scheme $X$ over a d.v.r. (i.e. a discrete valuation ring) $R$ of equal characteristic $p>0$ whith field of fractions $K$, such that the normal closure $\bar{Y}$ of $Y$ in $X$ does not have any structure of torsor which extends the one given on $Y$. Namely they construct such an example when $X=\operatorname{Spec}(R[x])$ and $G=\left(\mathbb{Z} / p^{2} \mathbb{Z}\right)_{K}$. Nevertheless one can ask whether we can find a scheme $Y^{\prime}$ and a torsor structure on it which extends the torsor structure on $Y$.

This problem is tightly related to the study of the fundamental group schemes of $X$ and of $X_{\eta}$. In [12] Nori gives the definition of the fundamental group scheme $\pi_{1}(X, x)$ of a reduced, connected and proper scheme $X$ over a perfect field $k$ provided with a point $x \in X(k)$. This definition has been extended by Gasbarri 
in [6] where he replaces $k$ by a Dedekind scheme $S$ (that is to say a normal noetherian scheme of dimension $\leq 1$ ) and where $X$ is a reduced and irreducible scheme faithfully flat over $S$. The two definitions coincide if $S$ is the spectrum of a perfect field.

In the first part of this paper we will briefly recall Nori's and Gasbarri's definitions of the fundamental group scheme and we state some preliminary lemmas necessary to solve our problem. Then we study the generic fiber of Gasbarri's fundamental group scheme of a scheme $X$ over a connected Dedekind scheme $S$ putting it in relation with the fundamental group scheme of $X_{\eta}$, the generic fiber of $X$. The principal results of this paper are theorems 2.2 and 3.1. In theorem 2.2 we prove that the natural morphism $\varphi: \pi_{1}\left(X_{\eta}, x_{\eta}\right) \rightarrow$ $\pi_{1}(X, x)_{\eta}$ is always faithfully flat. This has been recently proved by Garuti (cf. [5] §4, Theorem 4) when $X$ is normal and $S$ is the spectrum of a d.v.r.. As an application we prove theorem 3.1, that gives sufficient and necessary condition for a pointed torsor over $X_{\eta}$ to be extended over $X$. As a corollary we have that any dominated pointed torsor over $X_{\eta}$ (the meaning of "dominated torsor" will be explained in the text) can be extended to a pointed torsor over $X$ if and only if $\varphi$ is an isomorphism. This is always the case (see proposition 3.2) for $X$ an abelian scheme whose fundamental group scheme is isomorphic to the divisible group of $X$, i.e. the inverse limit of the kernels of the multiplication by an integer maps (cf. again proposition 3.2) using what Nori has already proved over a field (cf. [14]). In this case we prove that any pointed torsor (not only dominated) over the generic fiber $X_{\eta}$ of $X$ can be extended if and only if the finite group scheme acting on it has a model, which is always true when the field of functions of $S$ has characteristic 0 (cf. 3.3).

\section{The fundamental group scheme}

\subsection{Preliminaries}

In [12, Nori defines the fundamental group scheme $\pi_{1}(X, x)$ of a reduced, connected and proper scheme $X$ over a perfect field $k$ provided with a point $x \in X(k)$ as the group scheme associated to the neutral tannakian category $\left(E F(X), \otimes, x^{*}, \mathcal{O}_{X}\right)$ of essentially finite vector bundles over $X$. In [13], Part I, Ch. II, $\S 1$ Nori gives a second equivalent description for his fundamental group scheme. Gasbarri in [6] develops this point of view. We give some details on Gasbarri's construction. So from now on let $S$ be a Dedekind scheme, $X$ a reduced, irreducible scheme, $j: X \rightarrow S$ a faithfully flat morphism and $x: S \rightarrow X$ a fixed $S$-valued point.

Definition. Let $\mathcal{P}(X)$ be the category whose objects are triples $(Y, G, y)$ where:

1. $G$ is a finite and flat $S$-group scheme.

2. $f: Y \rightarrow X$ is a $G$-torsor for the fpqc topology.

3. $y: S \rightarrow Y$ is a section such that $f(y)=x$. 
A morphism $\varphi:\left(Y_{1}, G_{1}, y_{1}\right) \rightarrow\left(Y_{2}, G_{2}, y_{2}\right)$ between two triples is the datum of two morphisms $\alpha: Y_{1} \rightarrow Y_{2}$ and $\beta: G_{1} \rightarrow G_{2}$ where $\beta$ is a group scheme morphism, $\alpha\left(y_{1}\right)=y_{2}$ and such that the following diagram

$$
\begin{array}{ccc}
G_{1} \times Y_{1} & \rightarrow & Y_{1} \\
\downarrow & \circlearrowleft & \downarrow \\
G_{2} \times Y_{2} & \rightarrow & Y_{2}
\end{array}
$$

commutes, the horizontal arrows being the actions.

Lemma 2.1. Let $\alpha: G \rightarrow H$ be a group scheme morphism, $Y$ a G-torsor over $X, P$ an $H$-torsor over $X$ and $\varphi: Y \rightarrow P$ a morphism between torsors compatible with the actions of $G$ and $H$. Then $P$ is isomorphic to the contracted product $Y \times{ }^{G} H$ (as defined in [3], III, \$4, 3.2).

Proof. For any $X$-scheme $T$ we have a canonical arrow:

$$
\begin{array}{ccc}
Y(T) \times H(T) & \rightarrow & P(T) \\
(y, h) & \mapsto & \varphi(y) \cdot h
\end{array}
$$

that passes to quotient (under the left action of $G$ ). We deduce a morphism of $H$-torsors $Y \times{ }^{G} H \rightarrow P$ over $X$ which is then an isomorphism since every morphism between $H$-torsors is an isomorphism, hence the desired result.

Let $I$ be the set of isomorphism classes of objects of $\mathcal{P}(X)$; it is a poset when provided with the following relation: if $i, j \in I$ then $i \leq j$ if and only if there exists a morphism from the triple corresponding to $j$ to the triple corresponding to $i$. Moreover the following theorem holds:

Theorem 2.1. The set $I$ is filtered. Then we can define a pro-object $\mathrm{lim}_{i \in I}\left(Y_{i}, G_{i}, x_{i}\right)$. Moreover, $\pi_{1}(X, x)=\underline{\lim }_{i \in I} G_{i}$ is an S-group scheme and $\widetilde{\widetilde{Y}}=\lim _{i \in I} Y_{i}$ is a scheme.

Proof. See [6], Proposition 2.1.

Definition. We call the $S$-group scheme $\pi_{1}(X, x)$ constructed in theorem 2.1 the fundamental group scheme. We call the scheme $\tilde{Y}$ the $\pi_{1}(X, x)$-universal torsor over $X$.

Remark. When they can be compared Nori's and Gasbarri's construction coincide (cf. [13], Ch. II). Thus from now on we denote by $\pi_{1}(X, x)$ both Nori's and Gasbarri's fundamental group schemes and no confusion will arise.

Remark. There is a bijection between isomorphism classes of triples $(Y, G, y)$ as in def. 2.1 and $S$-group scheme morphisms $\rho: \pi_{1}(X, x) \rightarrow G$. Indeed given a triple $(Y, G, y)$, the morphism $\rho: \pi_{1}(X, x) \rightarrow G$ comes directly from theorem 2.1. On the other direction it is sufficient to consider the contracted product $\widetilde{Y} \times{ }^{\pi_{1}(X, x)} G$. Lemma 2.1 ensures that one direction is the inverse of the other. 


\subsection{Some elementary lemmas}

If not stated otherwise $S$ is any scheme. All group schemes that we will consider will be affine over $S$. We recall that a morphism of schemes $f: Z \rightarrow Y$ is called schematically dominant (cf. [10, Définition 11.10.2) if the corresponding morphism of $\mathcal{O}_{X}$-modules $f^{\#}: \mathcal{O}_{Y} \rightarrow f_{*}\left(\mathcal{O}_{Z}\right)$ is injective.

Remark. Let $\beta: G^{\prime} \rightarrow G$ be a morphism of affine (not necessarily finite) group schemes over a field. The following are equivalent:

1. $\beta: G^{\prime} \rightarrow G$ is schematically dominant,

2. $\beta: G^{\prime} \rightarrow G$ is surjective for the $f p q c$ topology,

3. $\beta: G^{\prime} \rightarrow G$ is faithfully flat.

Indeed 1) $\Leftrightarrow 2$ ) comes from [18], Ch. 15, §5 and 2) $\Leftrightarrow 3)$ [18, Ch. 14, §1.

Definition. Let $S$ be a Dedekind scheme. A triple $(Y, G, y)$, as defined before, is said to be a dominated triple 1 if for any triple $\left(Y^{\prime}, G^{\prime}, y^{\prime}\right)$ and any morphism $\varphi=(\alpha, \beta):\left(Y^{\prime}, G^{\prime}, y^{\prime}\right) \rightarrow(Y, G, y), \beta$ is a schematically dominant morphism. We will often refer to a triple (resp. dominated triple) $(Y, G, y)$ as a pointed (resp. dominated pointed) torsor.

For the sake of completeness we give some details for the easy proofs of the following useful lemmas.

Lemma 2.2. Any $S$-morphism $f: G^{\prime} \rightarrow G$ between group schemes can be factored into a schematically dominant morphism $s: G^{\prime} \rightarrow F$ (F some $S$-group scheme) and a closed immersion $i: F \hookrightarrow G$ such that $i \circ s=f$ :

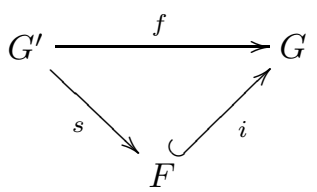

Proof. The question being local on $S$, we can assume $S$ to be an affine scheme, thus we set $S:=\operatorname{Spec}(B), G:=\operatorname{Spec}(C), G^{\prime}:=\operatorname{Spec}(A)$ to be affine. Denote by $h: A \rightarrow C$ the $B$-Hopf algebra morphism corresponding to $f$. Then $F:=$ $\operatorname{Spec}(\operatorname{Im}(h))$ is the $B$-group scheme with the desired properties. Indeed let $\Delta_{A}: A \rightarrow A \otimes A$ and $\Delta_{C}: C \rightarrow C \otimes C$ be the comultiplications of (resp.) $A$ and $C$. By the following commutative diagram:

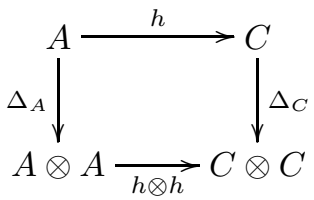

\footnotetext{
${ }^{1}$ N.B.: such a triple is called a "reduced triple" in [13], Part I, Ch. II when $S$ is the spectrum of a field. Because of the confusion that can arise we have decided to call it in a different manner.
} 
one deduces that $H:=\operatorname{Im}(h)$, the $B$-submodule image of $h$ (then $B$-flat as $C$ is), is a $B$-subcoalgebra of $C$ since $\Delta_{C}(H) \subseteq H \otimes H$. Similarly let $m_{A}: A \otimes A \rightarrow A$ and $m_{C}: C \otimes C \rightarrow C$ be the multiplications of $A$ and $C$ we have

$m_{C}(H \otimes H) \subseteq H$. If moreover $S_{A}$ and $S_{C}$ are the antipodal morphisms of $A$ and $C$ one can easily verify that $S_{C}(H) \subseteq H$ and this gives $H$ the desired $B$-Hopf algebra structure.

Lemma 2.3. Let $\left(G_{i}, \gamma_{i}^{l}\right)_{i \in I}$ be an inverse system of $S$-group schemes and $G=$ $\lim _{i \in I} G_{i}$; we have, for any pair $(i, l)$ such that $i \leq l$, the following commutative diagram:

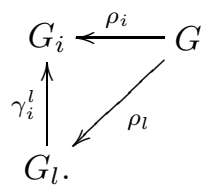

Then the canonical morphism $\rho_{i}$ is schematically dominant if and only if for any $l \geq i$ the map $\gamma_{i}^{l}: G_{l} \rightarrow G_{i}$ is schematically dominant.

Proof. As before we set $S:=\operatorname{Spec}(B), G_{i}:=\operatorname{Spec}\left(A_{i}\right)$ and $G:=\operatorname{Spec}(A)=$ $\operatorname{Spec}\left(\lim _{i \in I} A_{i}\right)$ and we prove the dual statement for $B$-Hopf algebras, where $f_{i}^{l}: A_{i} \rightarrow A_{l}$ corresponds to $\gamma_{i}^{l}$ and $\alpha_{i}: A_{i} \rightarrow A$ to $\rho_{i}$. One direction is obvious. In the other direction, we suppose that $f_{i}^{l}$ is injective for all $l \geq i$; let $x \in A_{i}$ and $\alpha_{i}(x)=0$. Now, we set $y:=f_{i}^{l}(x) \in A_{l}$, we know that $\alpha_{l}(y)=0$ according to the previous diagram; but $\alpha_{l}$ is defined as the composition of the following morphisms:

$$
\begin{array}{rllcc}
\alpha_{l}: \quad A_{l} & \hookrightarrow & \coprod_{k \in I} A_{k} & \rightarrow & \frac{\bigsqcup_{k \in I} A_{k}}{\sim} \simeq A \\
y & \mapsto y & \mapsto & 0
\end{array}
$$

where, for $a_{i} \in A_{i}$ and $a_{j} \in A_{j}$, we write $a_{i} \sim a_{j}$ if and only if there exists $k \geq i, k \geq j$ such that $f_{i}^{k}\left(a_{i}\right)=f_{j}^{k}\left(a_{j}\right)$ and this means that there exist $r \in I$, $r \geq l$ and $f_{l}^{r}: A_{l} \rightarrow A_{r}$ such that $f_{l}^{r}(y)=0$, in particular the morphism $f_{i}^{r}=f_{l}^{r} \circ f_{i}^{l}: A_{i} \rightarrow A_{r}$ maps $x$ into 0 , but according to the assumption on $A_{i}$ the morphism $f_{i}^{r}$ is injective and then $x=0$.

As a consequence we have the following

Corollary 2.1. Let $S$ be a Dedekind scheme. A triple $(Y, G, y)$ is dominated if and only if the morphism $\rho: \pi_{1}(X, x) \rightarrow G$ naturally associated to this triple is a schematically dominant morphism.

Lemma 2.4. Let $S$ be a Dedekind scheme. Let $\rho: \pi_{1}(X, x) \rightarrow G$ be an $S$ morphism of group schemes where $G$ is finite and flat over $S$. Then there exist an $S$-group scheme $G^{\prime}$ finite and flat over $S$, an $S$-morphism of group schemes $\rho^{\prime}: \pi_{1}(X, x) \rightarrow G^{\prime}$ and a closed immersion $\beta: G^{\prime} \rightarrow G$ such that $\beta \circ \rho^{\prime}=\rho$ and $\rho^{\prime}$ is a schematically dominant morphism. 
Proof. The existence of morphisms $\rho^{\prime}: \pi_{1}(X, x) \rightarrow G^{\prime}$ (schematically dominant) and $\beta: G^{\prime} \rightarrow G$ (closed immersion) such that $\beta \circ \rho^{\prime}=\rho$

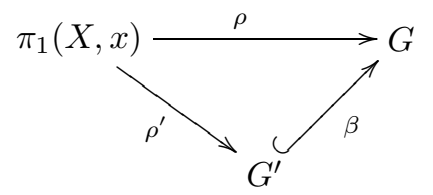

is ensured by lemma 2.2. The pointed torsor associated to $\rho^{\prime}$ is then dominated.

According to lemma 2.4 we can say that any triple $(Y, G, y)$ is preceded by a dominated triple or equivalently any pointed torsor over $X$ is preceded by a dominated pointed torsor.

Lemma 2.5. Let $\left(G_{i}, \gamma_{i}^{j}\right)_{i \in I}$ be an inverse system of $S$-group schemes, $G=$ $\lim _{i \in I} G_{i}$ and $\rho_{i}: G \rightarrow G_{i}$ the canonical morphism. Let $J$ be a filtered subset of $I$ and $G^{\prime}=\lim _{j \in J} G_{j}$. We assume that for any $j \in J$ the canonical morphism

$$
\rho_{j}^{\prime}: G^{\prime} \rightarrow G_{j}
$$

is schematically dominant. Then the natural morphism $\varphi: G \rightarrow G^{\prime}$ is schematically dominant if and only if $\rho_{j}: G \rightarrow G_{j}$ is schematically dominant for any $j \in J$.

Proof. Again we can assume that $S$ is affine, so we set $S:=\operatorname{Spec}(B), G_{i}:=$ $\operatorname{Spec}\left(A_{i}\right), G:=\operatorname{Spec}(A)=\operatorname{Spec}\left(\underline{\lim }_{i \in I} A_{i}\right)$ and $G^{\prime}:=\operatorname{Spec}(C)=\operatorname{Spec}\left(\underline{\lim }_{j \in J} A_{j}\right)$. For any $j \in J$ we have the following commutative diagram:

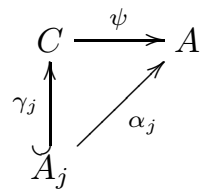

where $\psi, \alpha_{j}$ and $\gamma_{j}$ correspond respectively to $\varphi, \rho_{j}$ and $\rho_{j}^{\prime}$. If $\psi$ is injective then $\alpha_{j}$ is injective too for all $j \in J$ (obvious). Conversely, suppose $\alpha_{j}$ injective for all $j \in J$ and let $x \in C$ be such that $\psi(x)=0$. Once again we make use of the canonical factorisation:

$$
\gamma_{j}: A_{j} \hookrightarrow \coprod_{u \in J} A_{u} \rightarrow \frac{\coprod_{u \in J} A_{u}}{\sim} \simeq C .
$$

So let $z \in \coprod_{u \in J} A_{u}$ be a representing element of $x \in C$, it follows that there exists $v \in J$ such that $z \in A_{v}$ and $\gamma_{v}(z)=x$, in particular we have $0=\psi(x)=$ $\psi \circ \gamma_{v}(z)=\alpha_{v}(z)$, but since we have assumed $\alpha_{v}$ to be injective we have $z=0$, then $x=\gamma_{v}(z)=0$. 
Lemma 2.6. Let $\left(G_{i}, \gamma_{i}^{j}\right)_{i \in I}$ be an inverse system of $S$-group schemes, $G=$ $\lim _{i \in I} G_{i}$ and $\rho_{i}: G \rightarrow G_{i}$ the canonical morphism. Let $J$ be a filtered subset of $I$ and $G^{\prime}=\lim _{j \in J} G_{j}$. If for every $i \in I$ there exists a morphism $\gamma_{i}: G^{\prime} \rightarrow G_{i}$ such that the following diagram

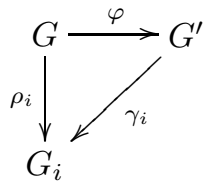

commutes, then $\varphi$ is a closed immersion. If moreover every $\rho_{i}$ is schematically dominant then $\varphi$ is an isomorphism.

Proof. Similar to the previous one.

\subsection{A comparison theorem}

From now on $S$ will denote a connected Dedekind scheme. We have recalled that the fundamental group scheme is the projective limit of $S$-finite and flat group schemes as follows

$$
\pi_{1}(X, x):=\underbrace{i \in I}_{\lim } G_{i}
$$

we will denote by

$$
\rho_{i}: \pi_{1}(X, x) \rightarrow G_{i}
$$

the corresponding canonical morphisms.

Proposition 2.1. Let $J \subseteq I$ be the set of all $i \in I$ such that $\rho_{i}: \pi_{1}(X, x) \rightarrow G_{i}$ is a schematically dominant morphism. The group scheme $\pi_{1}(X, x)$ is isomorphic to the projective limit of all the finite and flat $S$-group schemes $G_{j}, j \in J$, i.e. $\pi_{1}(X, x) \simeq \underline{\lim }_{j \in J} G_{j}$.

Proof. $J$ is filtered, indeed the construction made in [6], Proposition 2.1 still holds: so given two dominated pointed torsors $\left(Y_{1}, G_{1}, y_{1}\right)$ and $\left(Y_{2}, G_{2}, y_{2}\right)$ and morphisms over a third dominated pointed torsor $(Y, G, y)$ over $X$ we can construct a fourth pointed torsor $(P, H, p)$ with morphisms over the first two making the diagram

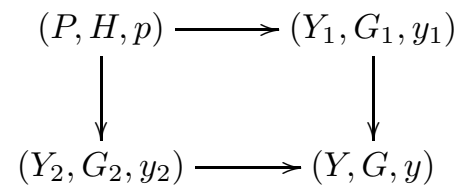

commute. If $(P, H, p)$ is not a dominated torsor it is sufficient to use lemma 2.4 in order to find a dominated pointed torsor $\left(P^{\prime}, H^{\prime}, p^{\prime}\right)$ making a similar diagram commute. Since $J \subset I$ we have a canonical morphism $v: \pi_{1}(X, x) \rightarrow$ $\lim _{j \in J} G_{j}$ which is an isomorphism: indeed it is schematically dominant by 
lemma 2.5, since by definition for all $j \in J \quad \pi_{1}(X, x) \stackrel{v}{\longrightarrow} l_{\longleftarrow} i_{j \in J} G_{j} \longrightarrow G_{j}$ is schematically dominant, and a closed immersion by lemma 2.6 since for all $i \in I$ there exists $j \in J$ such that the canonical morphism $\rho_{i}: \pi_{1}(X, x) \rightarrow G_{i}$ factors through $G_{j}$ hence we have a natural morphism $v_{i}: \lim _{j \in J} G_{j} \rightarrow G_{j} \rightarrow G_{i}$ such that $v_{i} \circ v=\rho_{i}$.

Now, let $\eta$ be the generic point of $S$, we construct $X_{\eta}:=X \times_{S} \eta$ that possesses a point $x_{\eta} \in X_{\eta}(\eta)$ (fiber of $x \in X(S)$ ). Over $\eta$ we can construct the fundamental group scheme

$$
\pi_{1}\left(X_{\eta}, x_{\eta}\right):=\lim _{m \in M} F_{m},
$$

where $M$ is the set of isomorphism classes of objects of $\mathcal{P}\left(X_{\eta}\right)$, but also the group scheme

$$
\pi_{1}(X, x)_{\eta}:=\pi_{1}(X, x) \times_{S} \eta .
$$

Now we compare the fundamental group scheme $\pi_{1}\left(X_{\eta}, x_{\eta}\right)$ and the generic fiber $\pi_{1}(X, x)_{\eta}$ of the fundamental group scheme of $X$.

Direct limits of algebras commute with base change (cf. [11], Appendix A, Theorem A.1), so the same is true for inverse limits of affine group schemes. Hence one gets

$$
\pi_{1}(X, x)_{\eta} \simeq \lim _{j \in J} G_{j, \eta}
$$

where $G_{j, \eta}:=G_{j} \times_{S} \eta$ and $\rho_{j, \eta}: \pi_{1}(X, x)_{\eta} \rightarrow G_{j, \eta}$ denotes the fiber of $\rho_{j}$. Since $\eta \rightarrow S$ is flat and $\rho_{j}$ is schematically dominant then $\rho_{j, \eta}$ is schematically dominant too. We consider the set $J^{\prime}:=J / \sim$ where for $j_{1}, j_{2} \in J$ we set $j_{1} \sim j_{2}$ if and only if $\rho_{j_{1}, \eta} \simeq \rho_{j_{2}, \eta}$; thus

$$
\pi_{1}(X, x)_{\eta} \simeq \underbrace{}_{\lim _{j \in J^{\prime}}} G_{j, \eta} .
$$

Lemma 2.7. $J^{\prime}$ is filtered and there is an injective map $J^{\prime} \hookrightarrow M$.

Proof. We are given two pointed torsors $\left(Y_{1, \eta}, G_{j_{1}, \eta}, y_{1, \eta}\right)$ and $\left(Y_{2, \eta}, G_{j_{1}, \eta}, y_{2, \eta}\right)$ and morphisms over a third pointed torsor $\left(Y_{\eta}, G_{j, \eta}, y_{\eta}\right)$ over $X_{\eta}$ (for $j, j_{1}, j_{2} \in$ $\left.J^{\prime}\right)$ then since $J \subset I$ is filtered there exists a triple $\left(Y^{\prime}, G^{\prime}, y^{\prime}\right)$ over $X$ dominating $\left(Y_{1}, G_{1}, y_{1}\right)$ and $\left(Y_{2}, G_{2}, y_{2}\right)$ whose generic fiber makes the obvious diagram commute.

As a consequence there exists a morphism

$$
\varphi: \pi_{1}\left(X_{\eta}, x_{\eta}\right) \longrightarrow \pi_{1}(X, x)_{\eta} .
$$

We can now state the principal result of this section:

Theorem 2.2. The morphism $\varphi: \pi_{1}\left(X_{\eta}, x_{\eta}\right) \longrightarrow \pi_{1}(X, x)_{\eta}$ is faithfully flat.

As a first application of this result we give a non trivial example where the fundamental group scheme is trivial. Thus in particular $\pi_{1}\left(\mathbb{P}_{S}^{1}, x\right)$ is trivial for $S$ a connected Dedekind scheme and $x \in \mathbb{P}_{S}^{1}(S)$ : 
Example. Let $S$ be a connected Dedekind scheme, $X$ an integral scheme, faithfully flat over $S$ and $x: S \rightarrow X$ a section. If the generic fiber of $X$ is a complete normal rational variety, then $\pi_{1}(X, x)$ is trivial

Proof. Let $\eta$ be the generic point of $S$. That $\pi_{1}\left(X_{\eta}, x_{\eta}\right)$ is trivial follows by [13, Ch II, Proposition 9 and its corollary). The fundamental group scheme $\pi_{1}(X, x)$ is flat over $S$ and has trivial generic fiber then it coincides with the scheme theoretic closure of $\{1\}_{\eta}$ in $\pi_{1}(X, x)$, which is then trivial.

Proof of Theorem. 2.2. We prove that

$$
\varphi: \pi_{1}\left(X_{\eta}, x_{\eta}\right) \longrightarrow \pi_{1}(X, x)_{\eta}
$$

is a schematically dominant morphism. For any $j \in J^{\prime}$ we consider the following commutative diagram:

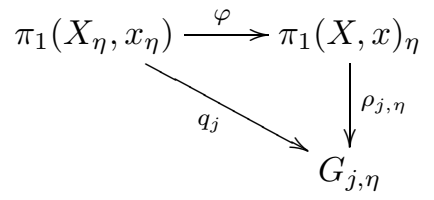

According to lemma 2.5 since $\rho_{j, \eta}: \pi_{1}(X, x)_{\eta} \rightarrow G_{j, \eta}$ is schematically dominant for any $j \in J^{\prime}$ (which is filtered in $M$, cf. lemma 2.7), it is sufficient to prove that for all $j \in J^{\prime}$ the morphism $q_{j}: \pi_{1}\left(X_{\eta}, x_{\eta}\right) \rightarrow G_{j, \eta}$ is schematically dominant too. By lemma 2.2 we split $q_{j}: \pi_{1}\left(X_{\eta}, x_{\eta}\right) \rightarrow G_{j, \eta}$ into a schematically dominant morphism followed by a closed immersion:

$$
q_{j}: \pi_{1}\left(X_{\eta}, x_{\eta}\right) \rightarrow G \hookrightarrow G_{j, \eta} .
$$

where $G$ is a finite $K$-group scheme.

Let $\left(Y^{\prime}, G, y^{\prime}\right)$ be the dominated pointed torsor associated to $\pi_{1}\left(X_{\eta}, x_{\eta}\right) \rightarrow G$ and let $\left(Y, G_{j, \eta}, y\right)$ be the pointed torsor associated to $q_{j}: \pi_{1}\left(X_{\eta}, x_{\eta}\right) \rightarrow G_{j, \eta}$. The latter is isomorphic to the contracted product (cf. lemma 2.1)

$$
Y \simeq Y^{\prime} \times{ }^{G} G_{j, \eta}
$$

via the morphism $f: G \hookrightarrow G_{j, \eta}$, and $y$ is the image in $Y$ of $y^{\prime}$. We immediately observe that the canonical morphism $f^{\prime}: Y^{\prime} \rightarrow Y$ is a closed immersion. Indeed locally, for the fpqc topology, it is certainly true since locally any torsor is trivial. We deduce that the result is also true globally since being a closed immersion is a local property for the $f p q c$ topology ([9], Proposition 2.7.1.). By construction there exist a finite and flat $S$-group scheme $G_{j}$ and a dominated triple $\left(P, G_{j}, p\right)$ over $X$ such that $\left(Y, G_{j, \eta}, y\right)$ is its generic fiber. The following diagram describes 
the present situation:

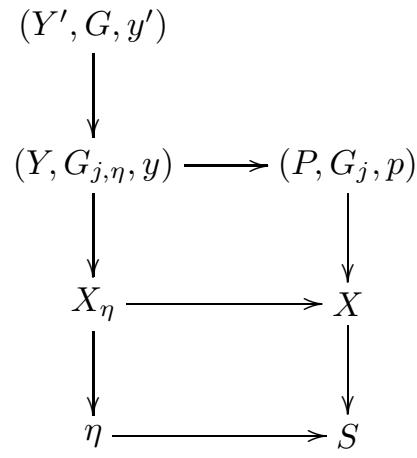

According to 9], proposition 2.8.5, there is a unique $S$-group scheme $H$, closed subgroup scheme of $G_{j}$ which is flat over $S$ and such that $H \times_{S} \eta \simeq G$ : it's the scheme theoretic closure of $G$ in $G_{j}$. Similarly we construct $Q$, the only closed subscheme of $P$ which is flat over $S$ and such that $Q \times_{S} \eta \simeq Y^{\prime}$. Again we construct the section $q: S \rightarrow Q$ as the scheme theoretic closure of $y^{\prime}$ in $p$. We have the following

Lemma 2.8. $(Q, H, q)$ is a pointed torsor over $S$.

Proof. (this is lemma 2.2 of [6] whose proof will be sketched here for the comfort of the reader) The scheme theoretic closure of closed subschemes of the generic fibre is fonctorial and commutes with fiber products (cf. [9], (2.8.3) and Corollaire 2.8.6) so in particular from diagram

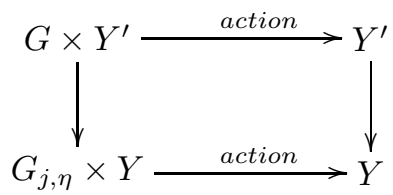

we deduce the commutative diagram

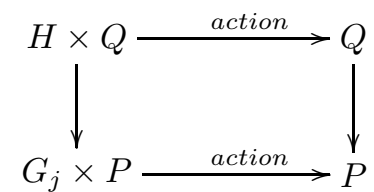

hence an action $H \times Q \rightarrow Q$. The isomorphism $G \times Y^{\prime} \simeq Y^{\prime} \times_{X_{\eta}} Y^{\prime}$ implies the isomorphism $\bar{G} \times \overline{Y^{\prime}} \simeq \overline{Y^{\prime} \times_{X_{\eta}} Y^{\prime}}$; the latter is the only closed subscheme of $P \times_{X} P$, flat over $S$ and whose generic fiber is isomorphic to $Y^{\prime} \times_{X_{\eta}} Y^{\prime}$. Since the same properties are satisfied by $Q \times_{X} Q$ then $\overline{Y^{\prime} \times_{X_{\eta}} Y^{\prime}} \simeq Q \times_{X} Q$ and consequently $H \times Q \simeq Q \times{ }_{X} Q$ and $Q$ is a $H$-torsor over $X$. With similar remarks we get the desired pointed torsor considering the section $q: S \rightarrow Q$. 
Thus we have a commutative diagram of triples:

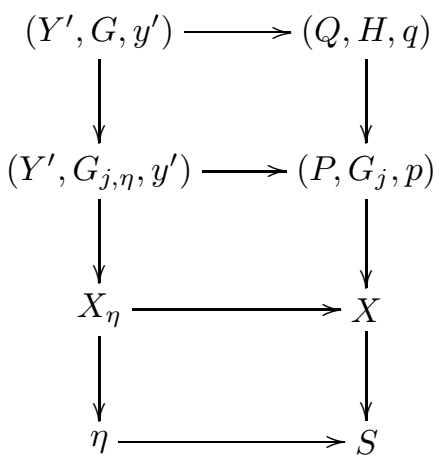

where the morphism $H \rightarrow G_{j}$ is by construction a closed immersion. But $\left(P, G_{j}, p\right)$ is a dominated pointed triple hence by definition the morphism $H \rightarrow$ $G_{j}$ is also schematically dominant then an isomorphism. So the same is true for the morphism $G \rightarrow G_{j, \eta}$, which proves that $q_{j}: \pi_{1}\left(X_{\eta}, x_{\eta}\right) \rightarrow G_{j, \eta}$ was already schematically dominant and this is enough to conclude.

Proposition 3.2 will provide an example where the morphism

$$
\varphi: \pi_{1}\left(X_{\eta}, x_{\eta}\right) \rightarrow \pi_{1}(X, x)_{\eta}
$$

is actually an isomorphism.

\section{Applications}

\subsection{Extension of torsors}

Now we apply theorem 2.2 to the problem of extending torsors or, more precisely, we explain how the kernel $N$ of the morphism $\varphi: \pi_{1}\left(X_{\eta}, x_{\eta}\right) \rightarrow \pi_{1}(X, x)_{\eta}$ measures the obstruction to extending a torsor over $X_{\eta}$ under the action of a finite group scheme to a torsor over $X$ under the action of a finite and flat $S$-group scheme; we fix some notations for this section:

Notation 3.1. From now on $S$ will be a connected Dedekind scheme, $\eta:=$ $\operatorname{Spec}(K)$ its function field, $X$ an integral scheme and $j: X \rightarrow S$ a faithfully flat morphism. We fix a section $x: S \rightarrow X$. We set $N:=\operatorname{ker}(\varphi)$ where $\varphi: \pi_{1}\left(X_{\eta}, x_{\eta}\right) \longrightarrow \pi_{1}(X, x)_{\eta}$ is the canonical morphism already described.

We prove the following

Theorem 3.1. Let $G$ be a finite group scheme over $K, \rho: \pi_{1}\left(X_{\eta}, x_{\eta}\right) \rightarrow G$ a schematically dominant morphism of $K$-group schemes and $(Y, G, y)$ the associated dominated pointed torsor. Then there exists a pointed torsor $\left(Y^{\prime}, G^{\prime}, y^{\prime}\right)$ over $X$, with $G^{\prime}$ a finite flat $S$-group scheme, whose generic fiber is isomorphic to $(Y, G, y)$ if and only if $N<\operatorname{ker}(\rho)$. 
A consequence of theorem 3.1 (and lemma 2.6) is the following

Corollary 3.1. Any dominated triple over $X_{\eta}$ can be extended to a (dominated) triple over $X$ if and only if $\varphi: \pi_{1}\left(X_{\eta}, x_{\eta}\right) \rightarrow \pi_{1}(X, x)_{\eta}$ is an isomorphism.

Proof of Theorem. 3.1. One direction is simple: assume in fact that there exists a triple $\left(Y^{\prime}, G^{\prime}, y^{\prime}\right)$ over $X$ whose generic fiber is isomorphic to $(Y, G, y)$. This means that there exists a morphism $\rho^{\prime}: \pi_{1}(X, x) \rightarrow G^{\prime}$ whose generic fiber $\rho_{\eta}^{\prime}: \pi_{1}(X, x)_{\eta} \rightarrow G^{\prime} \times_{S} \eta \simeq G$ satisfies $\rho_{\eta}^{\prime} \circ \varphi=\rho$, that is the following diagram commutes:

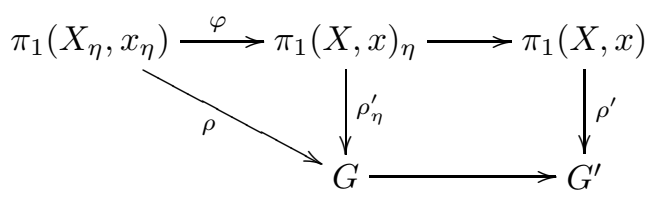

The existence of $\rho_{\eta}^{\prime}$ is equivalent (cf. [18, Ch. 15, Theorem 15.4) to the condition $N<\operatorname{ker}(\rho)$. Now, suppose that the condition $N<\operatorname{ker}(\rho)$ holds, then there exists a schematically dominant morphism $\gamma: \pi_{1}(X, x)_{\eta} \rightarrow G$ such that $\gamma \circ \varphi=\rho$, that is the following diagram commutes:

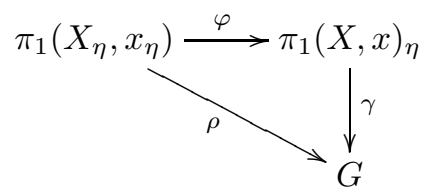

We recall that $\pi_{1}(X, x)_{\eta} \simeq \lim _{j \in J^{\prime}} G_{j, \eta}$ where $\rho_{j, \eta}: \pi_{1}(X, x)_{\eta} \rightarrow G_{j, \eta}$ is schematically dominant for all $j \in J^{\prime}$ (cf. isomorphism $(\dagger)$ ). Since to quotient $\pi_{1}(X, x)_{\eta}$, which is often not of finite type, by $N$ could be a problem we first need the following

Lemma 3.1. There exists $j \in J^{\prime}$ such that $\gamma$ factors through $G_{j, \eta}$, i.e. there exists a morphism $\gamma_{j}: G_{j_{\eta}} \rightarrow G$ such that the following diagram

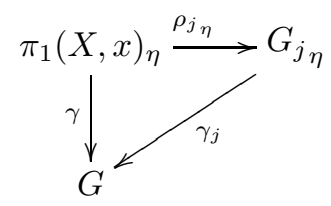

commutes.

Proof. This follows directly from the finiteness of $G$.

If $G$ is any $S$-group scheme and $H$ a closed subgroup scheme of $G$ we denote by $G / H_{(f p q c)}$ the sheaf associated, with respect to the fpqc topology, to the functor

$$
T \mapsto G(T) / H(T)
$$


from the category of schemes over $S$ to the category of sets. If $G / H_{(f p q c)}$ is represented by a $S$-scheme we denote it by $G / H$.

Proposition 3.1. Let $G$ and $H$ be two $S$-group schemes (here $S$ need not be a Dedekind scheme), $H \hookrightarrow G$ a closed immersion and assume that $G / H_{(f p q c)}$ is represented by a scheme $G / H$, then:

1. if $H$ is a normal closed subgroup scheme of $G$ then on $G / H$ there exists a unique structure of $S$-group scheme such that the canonical morphism $p: G \rightarrow G / H$ is a morphism of $S$-group schemes.

2. Let $T$ be any $S$-scheme and set $G_{T}:=G \times{ }_{S} T$ and $H_{T}:=H \times_{S} T$. Then $G_{T} / H_{T(f p q c)}$ is represented by the $T$-scheme $(G / H) \times_{S} T$.

3. the canonical morphism $p: G \rightarrow G / H$ is faithfully flat if and only if $H$ is flat over $S$.

Proof. Cf. 2], Proposition 9.2 (resp.) (iv), (v) and (xi).

Now we recall a particular case of [4], Théorème 7.1 which fits to our situation since finite implies projective (cf. [8] Corollaire 6.1.11):

Theorem 3.2. Let $S$ be any connected scheme. Let $G$ be a $S$-group scheme of finite type and let $H$ be a closed subgroup scheme of $G$, proper and flat over $S$. If $G$ is quasi projective over $S$ then $G / H_{(f p q c)}$ is representable.

Now we come back to the proof of theorem 3.1. The morphism $\gamma_{j}: G_{j, \eta} \rightarrow G$ from lemma 3.1 is schematically dominant since $\gamma$ is. We set

$$
N_{1}:=\operatorname{ker}\left(\gamma_{j}\right)
$$

which is a closed subgroup scheme of $N_{1}$. According to 9], Proposition 2.8.5, we construct the scheme theoretic closure of $N_{1}$ in $G_{j}$, that is an $S$-scheme $N_{2}$ which is the only closed subgroup scheme of $G_{j}$ flat over $S$ whose fibre is isomorphic to $G_{j, \eta}$. Moreover, according to [1, remarque 1.2.5.d), $N_{2}$ is normal in $G_{j}$. Let us denote by $G^{\prime}$ the $S$-quotient scheme $G_{j} / N_{2}$. Moreover, according to proposition 3.1, ii) there is an isomorphism $G \simeq G_{j, \eta} / N_{1} \simeq G^{\prime} \times_{S} \eta$. Then we have the following commutative diagram:

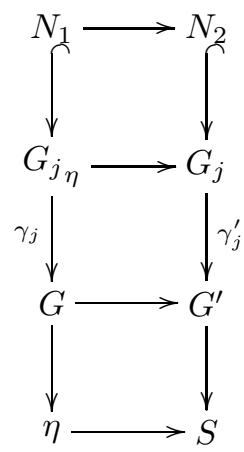


where we have denoted by $\gamma_{j}^{\prime}$ the morphism $G_{j} \rightarrow G^{\prime}$; we compose it with $\rho_{j}: \pi_{1}(X, x) \rightarrow G_{j}$ in order to obtain a morphism $\gamma_{j}^{\prime} \circ \rho_{j}: \pi_{1}(X, x) \rightarrow G^{\prime}$ to which we associate the triple $\left(Y^{\prime}, G^{\prime}, y^{\prime}\right)$ (cf. rem. 2.1) which is the desired triple. This concludes the proof of theorem 3.1

Now we explain how to extend torsors if they are related to other torsors that we know to be extensible. The proof of lemma 3.2 is similar to that of theorem 3.1 so we only sketch it.

Lemma 3.2. Let $(Y, G, y) \in \mathcal{P}(X)$ and $\left(Y_{\eta}, G_{\eta}, y_{\eta}\right)$ its generic fiber. Let $H^{\prime}$ be a $K$-group, $u: G_{\eta} \rightarrow H^{\prime}$ a faithfully flat morphism and $\left(Z^{\prime}, H^{\prime}, z^{\prime}\right)$ the associated object of $\mathcal{P}\left(X_{\eta}\right)$. Then there exists a triple $(Z, H, z) \in \mathcal{P}(X)$ whose generic fiber is isomorphic to $\left(Z^{\prime}, H^{\prime}, z^{\prime}\right)$.

Proof. Set $N:=\operatorname{ker}(u)$, then construct the scheme theoretic closure $\bar{N}$ of $N$ in $G$ and consider the quotient $H:=G / N$ in order to have the following diagram:

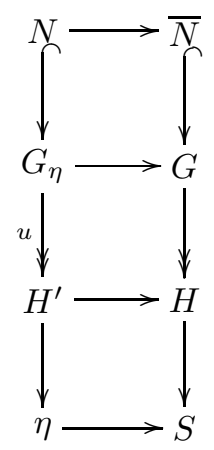

Roughly speaking this means that if we are able to extend a triple then we can extend any triple which is a "quotient" of the previous one (i.e. the morphism between their group schemes is faithfully flat). In the following lemma we solve a similar problem: suppose we are able to extend a triple, then we want to know whether we can extend a triple that "contains" it. More precisely:

Lemma 3.3. Let $(Y, G, y) \in \mathcal{P}(X)$ and $\left(Y_{\eta}, G_{\eta}, y_{\eta}\right)$ its generic fiber. Let $H^{\prime}$ be a $K$-group scheme such that $u: G_{\eta} \hookrightarrow H^{\prime}$ is a closed immersion and let $\left(Z^{\prime}, H^{\prime}, z^{\prime}\right)$ be the associated triple of $\mathcal{P}\left(X_{\eta}\right)$. Then there exists a triple $(Z, H, z) \in \mathcal{P}(X)$ whose generic fiber is isomorphic to $\left(Z^{\prime}, H^{\prime}, z^{\prime}\right)$ if and only if there exists a group scheme $L$ finite and flat over $S$ whose generic fiber is isomorphic to $H^{\prime}$.

Proof. If the triple $(Z, H, z) \in \mathcal{P}(X)$ exists just set $L:=H$. The other direction is the non abelian version of [15], Proposition 2.3.1 (a): we are in the following 
situation

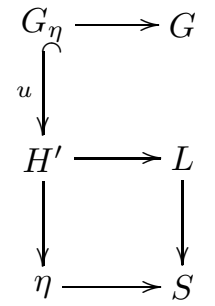

and we want to construct a $S$-finite and flat group scheme $H$ and a morphism $v: G \rightarrow H$ in order to obtain a cartesian diagram:

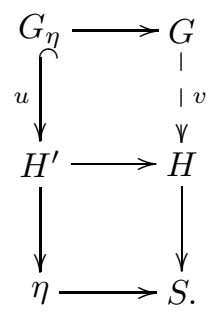

We can assume $S$ to be affine so set $S:=\operatorname{Spec}\left(R_{S}\right), G:=\operatorname{Spec}(A), L:=$ $\operatorname{Spec}(B), G_{\eta}:=\operatorname{Spec}\left(A_{\eta}\right)$ and $H^{\prime}:=\operatorname{Spec}\left(B_{\eta}\right)$ and consider the induced diagram

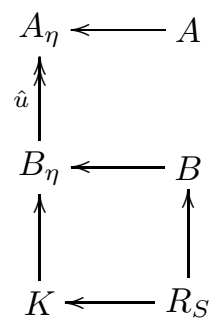

where $\eta:=\operatorname{Spec}(K)$. Now let $A^{\vee}$ and $B^{\vee}$ be the duals respectively of the commutative (but not necessarily cocommutative) Hopf $R_{S}$-algebras $A$ and $B$ : they are cocommutative (but not necessarily commutative and not necessarily Hopf) $R_{S}$-bialgebras; the antipodal morphisms $S_{A}: A \rightarrow A, S_{B}: B \rightarrow B$, which are morphisms of $R_{S}$-algebras, are transformed into $R_{S}$-coalgebra morphisms $S_{A}^{\vee}: A^{\vee} \rightarrow A^{\vee}, S_{B}^{\vee}: B^{\vee} \rightarrow B^{\vee}$. Similarly let $A_{\eta}^{\vee}$ and $B_{\eta}^{\vee}$ be the cocommutative $K$-bialgebras, duals respectively of $A_{\eta}$ and $B_{\eta}$, provided with the $K$-coalgebra morphisms $S_{A_{\eta}}^{\vee}: A_{\eta}^{\vee} \rightarrow A_{\eta}^{\vee}, S_{B_{\eta}}^{\vee}: B_{\eta}^{\vee} \rightarrow B_{\eta}^{\vee}$, then consider the diagram 


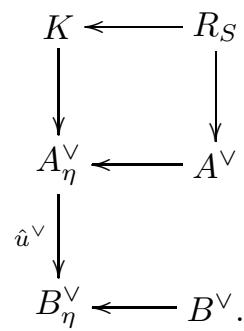

Now factor $\hat{u}^{\vee}=\varphi \circ \rho_{\eta}: A_{\eta}^{\vee} \rightarrow B_{\eta}^{\vee}$ where $\rho_{\eta}: A_{\eta}^{\vee} \rightarrow A_{\eta}^{\vee} \otimes B_{\eta}^{\vee}$ is the generic fiber of $\rho: A^{\vee} \rightarrow A^{\vee} \otimes B^{\vee}, x \mapsto x \otimes 1$ and $\varphi: A_{\eta}^{\vee} \otimes B_{\eta}^{\vee} \rightarrow B_{\eta}^{\vee}, x \otimes y \mapsto \hat{u}^{\vee}(x) \cdot y$ and consider the diagram

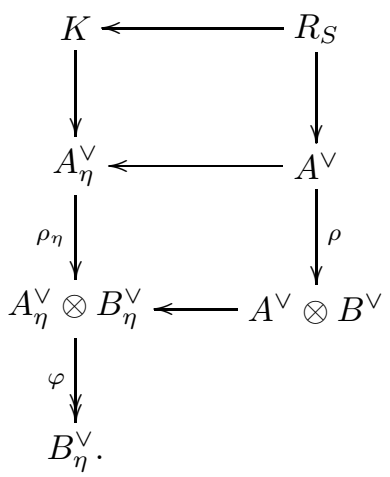

According to 9] (Proposition 2.8.1; (2.8.3); Proposition 2.8.4) we complete the previous diagram by constructing $\varphi^{\prime}: A^{\vee} \otimes B^{\vee} \rightarrow C$, where $C$ is the only $R_{S^{-}}$ flat module quotient of $A^{\vee} \otimes B^{\vee}$ whose generic fiber is isomorphic to $B_{\eta}^{\vee}$ (it is moreover a cocommutative bialgebra provided with a $R_{S}$-coalgebra morphism $\left.S_{C}: C \rightarrow C\right)$. Its dual $C^{\vee}$ is a commutative bialgebra, flat over $R_{S}$ whose generic fiber is isomorphic to $B_{\eta}$, and the dual morphism $S_{C}^{\vee}: C^{\vee} \rightarrow C^{\vee}$ gives $C^{\vee}$ a Hopf algebra structure; set $H:=\operatorname{Spec}\left(C^{\vee}\right)$, consider $\varphi^{\prime \vee}: C^{\vee} \rightarrow A \otimes B$ the dual morphism of $\varphi^{\prime}$ then the composition $\rho^{\vee} \circ \varphi^{\prime \vee}: C^{\vee} \rightarrow A$ induces a morphism of $S$-group schemes $\psi: G \rightarrow H$ which allows us to construct the desired triple $(Z, H, z)$ as the contracted product of $(Y, G, y)$ via $\psi$.

Remark. The assumption that $u: G_{\eta} \rightarrow H^{\prime}$ is a closed immersion is never used, but it is the only case of interest in our situation according to lemma 2.2

Remark. When $\operatorname{char}(K)=0$ a group scheme $H$ as in the statement of Lemma 3.3 always exists.

\subsection{The case of an abelian scheme}

Let $S$ be any connected Dedekind scheme and assume that $X \rightarrow S$ is an abelian scheme (i.e. $X$ is a smooth and proper $S$-group scheme with geometric connected fibers), let $0_{X}$ be the unity for the group law of $X$ and for any natural 
number $m$ let $m_{X}: X \rightarrow X$ denote the multiplication by $m$. One observes that $\left(X,{ }_{m} X, 0_{X}\right)$ is a triple over $X$ where ${ }_{m} X:=\operatorname{ker}\left(m_{X}\right)$. In [14] Nori proves that for any point $s \in S$ and for any triple $\left(Y^{\prime}, G^{\prime}, y^{\prime}\right) \in \mathcal{P}\left(X_{s}\right)$ there exists a natural number $n$ and

1. a morphism of group schemes $u:{ }_{n}\left(X_{s}\right) \rightarrow G^{\prime}$,

2. a morphism $X_{s} \rightarrow Y^{\prime}$ commuting with the actions of ${ }_{n}\left(X_{s}\right)$ and $G^{\prime}$

hence in particular, $\pi_{1}\left(X_{s}, 0_{X_{s}}\right) \simeq \lim _{n}\left({ }_{n}\left(X_{s}\right)\right)$. It is clear that the triple $\left(X_{s},{ }_{n}\left(X_{s}\right), 0_{X_{s}}\right)$ is isomorphic to the fiber in $s$ of $\left(X,{ }_{n} X, 0_{X}\right)$ then we have the following

Proposition 3.2. Let $X$ be an abelian scheme over a connected Dedekind scheme $S$, then for every $s \in S$ the canonical morphism

$$
\varphi: \pi_{1}\left(X_{s}, x_{s}\right) \rightarrow \pi_{1}(X, x)_{s}
$$

is an isomorphism. Moreover $\pi_{1}\left(X, 0_{X}\right) \simeq \lim _{n}\left({ }_{n} X\right)$.

Proof. Every triple $\left(Y_{s}, G_{s}, y_{s}\right)$ over $X_{s}$ fiber of a triple $(Y, G, y)$ over $X$ is preceded by a triple $\left(X_{s},{ }_{n}\left(X_{s}\right), 0_{X_{s}}\right)$ for a certain $n$ then every arrow $\pi_{1}\left(X, 0_{X}\right)_{s} \rightarrow$ $G_{s}$ factors through ${ }_{n}\left(X_{s}\right)$ hence the first assertion. Thus for any $s \in S$ the fiber $\psi_{s}: \pi_{1}\left(X, 0_{X}\right)_{s} \rightarrow \lim _{n}\left({ }_{n}\left(X_{s}\right)\right)$ is an isomorphism. As usual let $\eta$ be the generic point of $S$ and consider the canonical morphism $\psi: \pi_{1}\left(X, 0_{X}\right) \rightarrow \lim _{n}\left({ }_{n} X\right)$. Assume for a moment that it is faithfully flat, then it is sufficient to consider its kernel which is flat and then trivial since it coincides with the scheme theoretic closure of $\{1\}_{\eta}$, the kernel of $\psi_{\eta}$, in $\pi_{1}\left(X, 0_{X}\right)$. In order to prove it is faithfully flat then one has to show that for any $n \in \mathbb{N}$ the canonical morphism $\pi_{1}\left(X, 0_{X}\right) \rightarrow{ }_{n} X$ is faithfully flat too. To show this, considering the usual projective limit $\pi_{1}\left(X, 0_{X}\right)=\lim _{i \in I} G_{i}$, it is sufficient to prove that whenever there is a morphism $G_{i} \rightarrow{ }_{n} X$ then it is faithfully flat. But this is certainly true on the fibers, since $\left(X_{s},{ }_{n}\left(X_{s}\right), 0_{X_{s}}\right)$ is a dominated triple for any $s \in S$ then $\left(G_{i}\right)_{s} \rightarrow{ }_{n}\left(X_{s}\right)$ is faithfully flat. Thus $G_{i} \rightarrow{ }_{n} X$ is faithfully flat by means of [10] Théorème 11.3.10. Hence $\psi$ is an isomorphism according to [3], $\mathrm{I}, \S 2, \mathrm{n}^{\circ} 3$, Corollaire 2.9.

Remark. That $\pi_{1}\left(X, 0_{X}\right)$ is isomorphic to $\lim _{n}\left({ }_{n} X\right)$ can be proven directly without considering fibers, following what Nori did in [14. We cannot use [10] Théorème 11.3.10 for $\psi$ because in general the fundamental group scheme is not of finite type.

Let $\eta$ be the generic point of $S$, if $\left(Y^{\prime}, G^{\prime}, y^{\prime}\right)$ is a dominated triple over $X$ then there exists $n \geq 1$ such that $u:{ }_{n}\left(X_{s}\right) \rightarrow G^{\prime}$ is faithfully flat and then, according to lemma 3.2, there exists a triple $(Y, G, y)$ extending $\left(Y^{\prime}, G^{\prime}, y^{\prime}\right)$. Then we have the following 
Proposition 3.3. When $X$ is an abelian scheme over a connected Dedekind scheme $S$, then every dominated triple $\left(Y^{\prime}, G^{\prime}, y^{\prime}\right) \in \mathcal{P}\left(X_{\eta}\right)$ can be extended to a triple $(Y, G, y) \in \mathcal{P}(X)$; moreover every non dominated triple $\left(Y^{\prime}, G^{\prime}, y^{\prime}\right)$ can be extended to a triple $(Y, G, y)$ if and only if there exists a finite and flat $S$-group scheme $H$ whose generic fiber is isomorphic to $G^{\prime}$.

Proof. That every dominated triple can be extended follows from previous discussion. Then apply lemma 3.3 to obtain the statement on non dominated triples.

Acknowledgements. This paper is part of my $\mathrm{PhD}$ thesis. I would like to thank my advisor Michel Emsalem for his guidance and his constant encouragement. This work has been partially supported by the Università degli Studi di Milano. I also would like to thank Matthieu Romagny, Carlo Gasbarri and Dajano Tossici for useful comments and discussions. Finally I would like to thank an anonymous referee for his suggestions, corrections and some improvements.

\section{References}

[1] S. Anantharaman, Schémas en groupes, espaces homogènes et espaces algébriques sur une base de dimension 1. Mémoires de la S. M. F., tome 33, 5-79 (1973).

[2] J. E. Bertin, Généralités sur les préschémas en groupes. éxposé $\mathrm{VI}_{B}$, Séminaires de Géométrie Algébrique Du Bois Marie. III , (1962/64)

[3] M. Demazure, P. Gabriel, Groupes algébriques, North-Holland Publ. Co., Amsterdam, (1970).

[4] P. Gabriel, Construction de préschémas quotient. Éxposé V, Séminaires de Géométrie Algébrique Du Bois Marie. III , (1962/64)

[5] C. Garuti, Barsotti-Tate Groups and p-adic Representations of the Fundamental Group Scheme, Math. Ann. 341, No. 3, 603-622 (2008).

[6] C. Gasbarri, Heights of Vector Bundles and the Fundamental Group Scheme of a Curve, Duke Math. J. 117, No.2, 287-311 (2003).

[7] A. Grothendieck, Éléments de géométrie algébrique. I. Le langage des schémas. Publications Mathématiques de l'IHÉS, 4, (1960).

[8] A. Grothendieck, Éléments de géométrie algébrique. II. Étude globale élémentaire de quelques classes de morphismes. Publications Mathématiques de l'IHÉS, 8, (1961).

[9] A. Grothendieck, Éléments de géométrie algébrique. IV. Étude locale des schémas et des morphismes de schémas. II, Publications Mathématiques de l'IHÉS, 24, (1965). 
[10] A. Grothendieck, Éléments de géométrie algébrique. IV. Étude locale des schémas et des morphismes de schémas. III, Publications Mathématiques de l'IHÉS, 28, (1966).

[11] H. Matsumura, Commutative Ring Theory, Cambridge University Press, (1980)

[12] M. V. NorI, On the Representations of the Fundamental Group, Compositio Matematica, Vol. 33, Fasc. 1, (1976). p. 29-42.

[13] M. V. Nori, The Fundamental Group-Scheme, Proc. Indian Acad. Sci. (Math. Sci.), Vol. 91, Number 2, (1982), p. 73-122.

[14] M. V. NorI, The Fundamental Group-Scheme of an Abelian Variety, Math. Ann. 263, (1983), p. 263-266.

[15] M. Raynaud, Schémas en groupes de type $(p, \ldots, p)$, Bulletin de la Société Mathématique de France, 102 (1974), p. 241-280.

[16] M. Romagny, Effective Models of Group Schemes, arXiv:0904.3167v2, (2009).

[17] M. SAIDI, Cyclic p-Groups and Semi-Stable Reduction of Curves in Equal Characteristic $p>0$, arXiv:math/0405529 (2004).

[18] W. C. Waterhouse, Introduction to Affine Group Schemes, GTM, Springer-Verlag, (1979).

Marco Antei

Laboratoire Paul Painlevé, U.F.R. de Mathématiques Université des Sciences et des Techonlogies de Lille 1 59655 Villeneuve d'Ascq

E-mail:

antei@math. univ-lille1.fr

marco.antei@gmail.com 